\title{
Stimulated Raman adiabatic passage from atomic to molecular Bose-Einstein condensates: Feedback laser-detuning control and suppression of dynamical instability
}

\author{
Jing Cheng, ${ }^{1,2}$ Shensheng Han, ${ }^{1}$ and YiJing Yan ${ }^{2}$ \\ ${ }^{1}$ Key Laboratory for Quantum Optics and Center for Cold Atom Physics, Shanghai Institute of Optics and Fine Mechanics, \\ Chinese Academy of Sciences, Shanghai 201800, China \\ ${ }^{2}$ Department of Chemistry, Hong Kong University of Science and Technology, Kowloon, Hong Kong
}

(Received 16 September 2005; published 30 March 2006)

\begin{abstract}
We present a feedback control scheme that designs time-dependent laser-detuning frequency to suppress possible dynamical instability in coupled free-quasibound-bound atom-molecule condensate systems. The proposed adaptive frequency chirp with feedback is shown to be highly robust and very efficient in the passage from an atomic to a stable molecular Bose-Einstein condensate.
\end{abstract}

DOI: 10.1103/PhysRevA.73.035601

PACS number(s): 03.75.Mn, 05.30.Jp, 32.80.Qk

Coherent conversion of an atomic Bose-Einstein condensate $(\mathrm{BEC})$ to a molecular BEC is an active research topic in cold atom physics [1-6]. Creating stable molecular condensates is important for studying the quantum superchemistry $[7,8]$, and producing atomic lasers. Both the Feshbach resonance $[1,2]$ and photoassociation $[3,4]$ have been proposed to produce a molecular condensate. However, the created molecules are generally in an excited quasibound state [9], often with a limited conversion efficiency to the desired stable (e.g., ground) state of molecular condensates. Two-photon Raman photoassociation transition to create a high density of stable molecules in a Mott insulator state has been observed recently [6]. The stimulated Raman adiabatic passage (STIRAP) [10] technique has also been suggested to convert cold atoms to molecules in a deeply bound ground state [11-16]. In a linear three-level $\Lambda$ system there exists the coherent population trapping (CPT) state under the twophoton resonance condition, and the STIRAP can be successfully realized by using the counterintuitive sequence of laser pulses [10]. As it is formed by the free atomic, the quasibound, and the ground molecular condensates, the condensate $\Lambda$ system experiences nonlinear shifts due to collisions between particles. These nonlinear shifts move the system off the required two-photon resonance in the conventional STIRAP scheme, and thus reduce the conversion efficiency.

Recently, Ling et al. [17] proposed a generalized STIRAP scheme by including a temporal evolution of laser frequency (i.e., frequency chirp) to compensate the collision-induced shifts dynamically. The two-photon resonance requirement can thus be fulfilled and high conversion efficiency is possible. However, the collision-embedded CPT states obtained in their scheme may be dynamically unstable in certain parameter regions. The purpose of this work is to show that this dynamical instability can be completely suppressed by introducing a feedback control to design the laser frequency chirp. Consequently, high-efficiency atom-molecule BEC conversions can be achieved robustly with the feedback STIRAP control in a broad parameter region.

Our model system shown in Fig. 1 is the same as Ref. [17], where the energy levels of free atomic, quasibound molecular, and ground state molecular condensates are $|a\rangle,|m\rangle$, and $|g\rangle$, respectively. States $|a\rangle$ and $|m\rangle$ are coupled via a magnetic field through Feshbach resonance with coupling strength $\alpha^{\prime}$ (or density scaled $\alpha \equiv \alpha^{\prime} \sqrt{n}$ ) and by detuning $\epsilon$, and $|m\rangle$ and $|g\rangle$ are coupled via a laser field with Rabi frequency $\Omega$ and detuning $\Delta$. As Ref. [17], we also treat the mean-field dynamics of matter amplitudes,

$$
\frac{d \psi_{a}}{d t}=-i\left(\lambda_{a}\left|\psi_{a}\right|^{2}+\lambda_{a m}\left|\psi_{m}\right|^{2}+\lambda_{a g}\left|\psi_{g}\right|^{2}\right) \psi_{a}-i \alpha \psi_{m} \psi_{a}^{*},
$$

$$
\begin{gathered}
\frac{d \psi_{m}}{d t}=-i\left(\lambda_{m}\left|\psi_{m}\right|^{2}+\lambda_{a m}\left|\psi_{a}\right|^{2}+\lambda_{m g}\left|\psi_{g}\right|^{2}\right) \psi_{m}-i \frac{\alpha}{2} \psi_{a}^{2}+i \frac{\Omega}{2} \psi_{g} \\
-(\gamma+i \epsilon) \psi_{m}, \\
\frac{d \psi_{g}}{d t}=-i\left(\lambda_{g}\left|\psi_{g}\right|^{2}+\lambda_{a g}\left|\psi_{a}\right|^{2}+\lambda_{m g}\left|\psi_{m}\right|^{2}\right) \psi_{g}+i \frac{\Omega}{2} \psi_{m} \\
-i(\Delta+\epsilon) \psi_{g},
\end{gathered}
$$

with the two-body collision coefficients $\lambda_{i}=4 \pi \hbar a_{i} n / m_{i}$ and $\lambda_{i j}=2 \pi \hbar a_{i j} n / m_{i j}$. Here $a_{i}\left(a_{i j}\right)$ is the $s$-wave scattering length, $m_{i}\left(m_{i j}\right)$ the mass (reduced mass), and $n$ the density of the total particle number. Introduced in Eq. (1b) is also a phenomenological loss parameter $\gamma$ for the decay of the quasibound molecules; thus $\left|\psi_{a}\right|^{2}+2\left|\psi_{m}\right|^{2}+2\left|\psi_{g}\right|^{2} \leqslant 1$. The equal sign holds, i.e., that the total particle number is conserved, when $\gamma=0$. Equations (1) serve as the starting point of many theoretical investigations on the formation of stable molecule condensates [15-17].

It has been shown that the steady states $\left\{\psi_{\nu}(t)\right.$ $\left.=\left|\psi_{\nu}^{0}\right| e^{i \theta_{\nu}} e^{-i \mu_{\nu} t} ; \nu=a, m, g\right\}$ of Eqs. (1) (with $\gamma=0$ and timeindependent $\Omega$ ) support a CPT solution under the laser detuning condition [17],

$$
\Delta=-\epsilon+\left(2 \lambda_{a g}-\lambda_{g}\right)\left|\psi_{g}^{0}\right|^{2}+\left(2 \lambda_{a}-\lambda_{a g}\right)\left|\psi_{a}^{0}\right|^{2},
$$

with the steady CPT state populations $\left|\psi_{m}^{0}\right|^{2}=0$ and 


$$
\left|\psi_{a}^{0}\right|^{2}=1-2\left|\psi_{g}^{0}\right|^{2}=\frac{2}{\sqrt{1+8(\alpha / \Omega)^{2}}+1},
$$

the phases $\theta_{g}=\theta_{m}=2 \theta_{a}$, and the chemical potentials $\mu_{g}=\mu_{m}$ $=2 \mu_{a}=2\left(\lambda_{a}\left|\psi_{a}^{0}\right|^{2}+\lambda_{a g}\left|\psi_{g}^{0}\right|^{2}\right)$. The existence of CPT solutions suggests the possibility of the STIRAP approach to atommolecule conversion, in which $\alpha / \Omega$ varies smoothly from 0 to $\infty$ (due to the counterintuitive pulses sequence), and the system follows the CPT states of Eq. (3) adiabatically from $\left|\psi_{a}(0)\right|^{2}=1$ to $\left|\psi_{g}(\infty)\right|^{2}=0$. To achieve that, the authors of Ref. [17] proposed to design the time-dependent laser detuning $\Delta(t)$ (or frequency chirp) via the direct substitution of Eq. (3) into Eq. (2). This is the direct control scheme that relates the time-dependent laser detuning $\Delta(t)$ a priori to the laser pulse field coupling $\Omega(t)$. However, this scheme suffers dynamical instability in certain regions in $(\Omega, \epsilon)$ space, called hereafter the Ling-Pu-Seaman (LPS) unstable region after the authors of Ref. [17]. The dynamical instability is uncontrollable with the direct control scheme as the laser detuning there does not cope with any unavoidable small fluctuation in the BEC system. This small initial uncertainty would then lead the system evolution deviating significantly from the required CPT solutions [cf. Fig. 2(a)].

To suppress the dynamical instability one shall relate the control field detuning $\Delta(t)$ explicitly to the system evolution, while retain its stationary CPT limit as Eq. (2). Actually, the last two terms of Eq. (2) arising from the nonlinear collision do suggest some possible schemes of feedback laserdetuning control on the atom-molecule conversion. After numerical testing on several possible versions, we propose here

$$
\Delta(t)=-\epsilon+\frac{1}{2}\left(2 \lambda_{a g}-\lambda_{g}\right)\left[1-\left|\psi_{a}(t)\right|^{2}\right]+\left(2 \lambda_{a}-\lambda_{a g}\right)\left|\psi_{a}(t)\right|^{2},
$$

which requires only the feedback of the time-dependent atomic condensate population. We shall show that not only the adaptive laser-detuning feedback control described above completely suppresses the dynamical instability in the CPT state, it is also very robust, resulting in high efficiency of coherent atom-molecule conversion. In the following demonstrations, we consider the model sodium BEC system used of Ref. [17], in which $\alpha=9.436 \times 10^{4} \mathrm{~s}^{-1}, \lambda_{a}=0.625 \alpha, \lambda_{m}=\lambda_{g}$ $=\lambda_{a m}=\lambda_{m g}=\lambda_{a g}=0.1875 \alpha$, and $\gamma=1.0 \alpha$.

Let us start with the stability analysis. Reported in Fig. 2 are the results on the CPT state, with the fixed values of $\Omega$ $=1.0 \alpha$ and $\epsilon=3 \alpha$ chosen from the LPS unstable region [17], against initial $0.1 \%$ population fluctuations. As shown in Fig. 2(a), the CPT state under the direct control scheme [Eq. (2)]

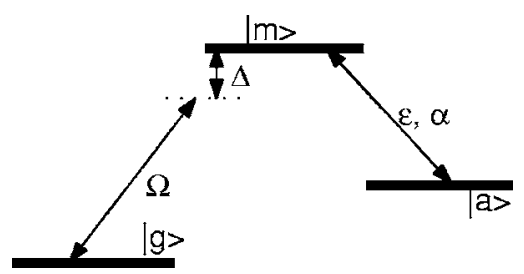

FIG. 1. Schematic diagram of three-state free-quasibound-bound atom-molecule system.

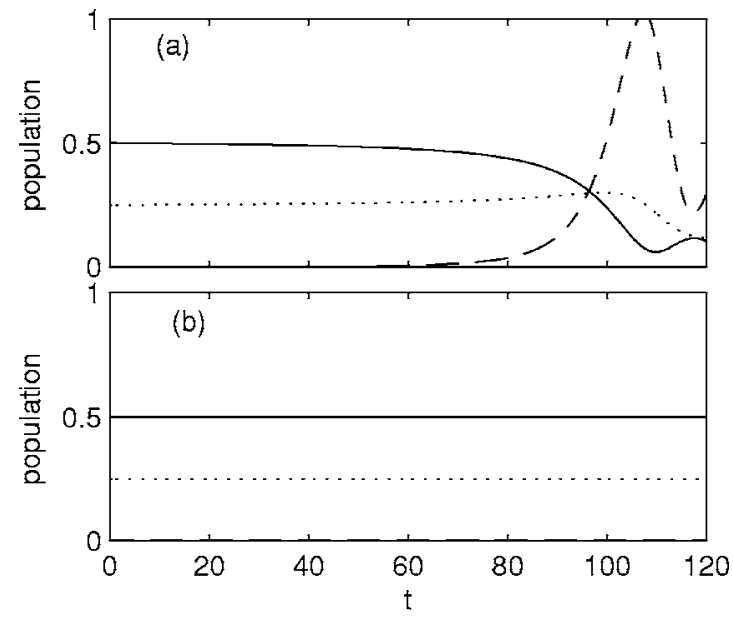

FIG. 2. Evolution of the CPT state populations in the LPS unstable region with initial $0.1 \%$ fluctuations: $\left|\psi_{a}(t)\right|^{2}$ (solid curves), $\left|\psi_{m}(t)\right|^{2} \times 100$ (dashed-curves), and $\left|\psi_{g}(t)\right|^{2}$ (dotted curves); (a) direct control scheme [Eq. (2)]; (b) feedback control scheme [Eq. (4)]. See parameters in text. The unit of $t$ is $\alpha^{-1}$.

is unstable; a small fluctuation would deviate it dynamically far away from the desired CPT state. However, with the help of the present feedback control scheme [Eq. (4)], as shown in Fig. 2(b), the fluctuations will not be amplified; the system is stable and does remain in the CPT state. We have also performed many other simulations, and found that for any values of $(\Omega, \epsilon)$ parameters, the CPT states are stable in the present feedback control scheme.

The above analysis suggests the possibility of a feedback STIPAP scheme for high-efficiency atom-molecule conversion. The system is initially in the atomic condensate state, and the conversion efficiency is defined as $\eta \equiv 2\left|\psi_{g}(\infty)\right|^{2}$. In the following simulations, the Rabi frequency of the Stokes laser field assumes

$$
\Omega(t)=\frac{\Omega_{\max }}{2}\left[1-\tanh \left(\frac{t-t_{0}}{\tau}\right)\right] .
$$

Unless further specification, $\Omega_{\max }=40 \alpha, \tau=40 / \alpha$, and $t_{0}$ $=120 / \alpha$, and the Feshbach field detuning $\epsilon=3 \alpha$. Note that the system will evolve into the LPS unstable region when $|\Omega(t)|$ becomes small [17]. The laser detuning $\Delta(t)$ as a function of time in the feedback control scheme [Eq. (4)] shall be evaluated with the system propagation [Eq. (1)], while in the direct control scheme it is independent of the system evolution and could have been determined by substituting Eqs. (5) and (3) into Eq. (2).

Shown in the top two panels of Fig. 3 are the resulting populations $\left|\psi_{a, m, g}(t)\right|^{2}$, as functions of time in the direct and feedback control schemes, respectively. Clearly, the direct method leads to a dynamical instability as the system is bound to evolve into the LPS unstable region after the peak laser power, resulting in a small conversion efficiency. Our feedback control scheme efficiently suppresses this kind of instability, achieving a high conversion efficiency of $\eta$ $=83 \%$. The bottom panel of this figure depicts the laser- 


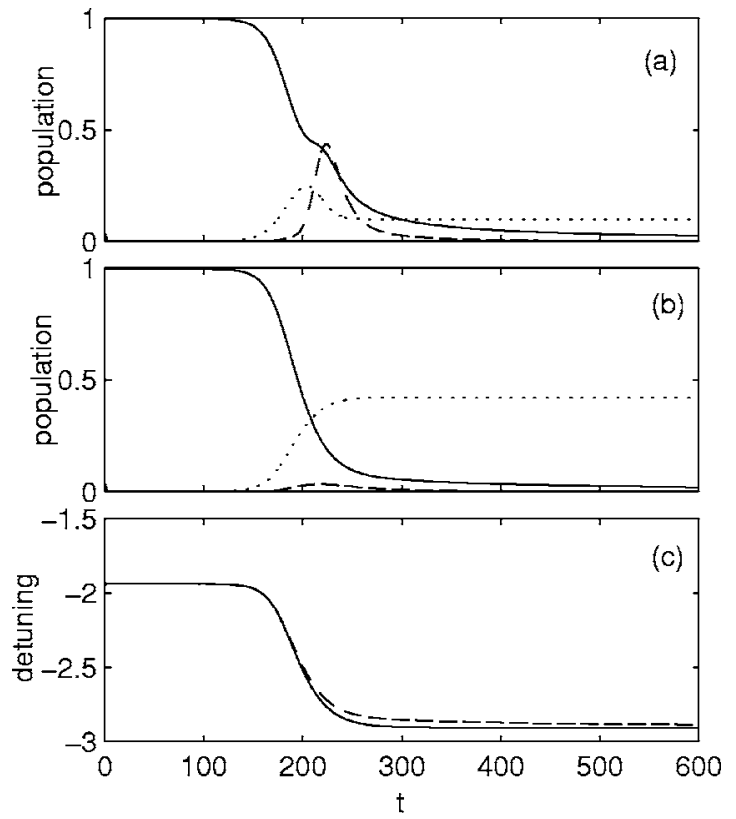

FIG. 3. (a) Evolution of populations, $\left|\psi_{a}(t)\right|^{2}$ (solid), $\left|\psi_{m}(t)\right|^{2}$ $\times 100$ (dashed), and $\left|\psi_{g}(t)\right|^{2}$ (dotted), from the direct control scheme. See text for the parameters. (b) Same as (a) but from the feedback control scheme. (c) Time-dependent laser detuning $\Delta(t)$; solid curve, via Eq. (2) and used in (a); dashed-curve, via Eq. (4) and used in (b). The unit of $t$ is $\alpha^{-1}$.

detuning $\Delta(t)$ functions associating with the above two control schemes. The difference between the two curves there seems small. It is this small difference in the time-dependent laser detuning, which changes completely the nature of atom-molecule conversion dynamics from unstable to stable. More importantly, the sensitivity described here does not deteriorate the robustness of the feedback control method. Reported in Fig. 4 is a robustness test on the feedback control, in which the adaptive $\Delta(t)$ [the dashed-curve in Fig. 3(c)] is subject to a small (2\% in relative amplitude) white-noise fluctuation $\delta \Delta(t)$ that models experimental uncertainty. Our feedback control method is shown to be very robust against small white-noise fluctuations.

In Fig. 5, we report the feedback control conversion efficiency diagram in the $\left(\Omega_{\max }, \epsilon\right)$ space. The observed high conversion efficiency $(\eta>75 \%)$ covers practically the entire STIRAP parameter region, where the Stokes laser coupling

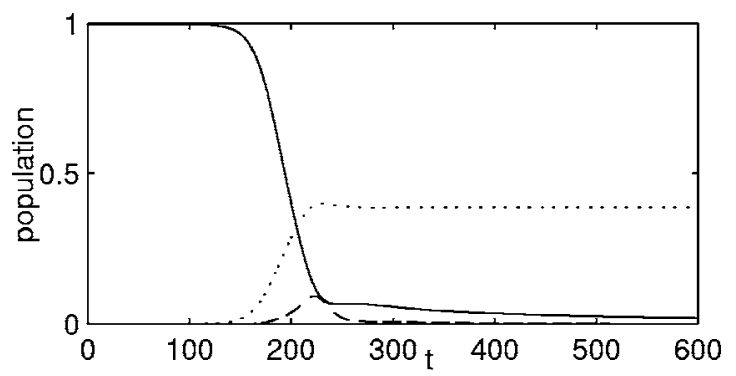

FIG. 4. Same as Fig. 3(b), but the adaptive laser detuning $\Delta(t)$ [dashed-curve in 3(c)] of the feedback control field is now subject to $2 \%$ white-noise fluctuation. The unit of $t$ is $\alpha^{-1}$.

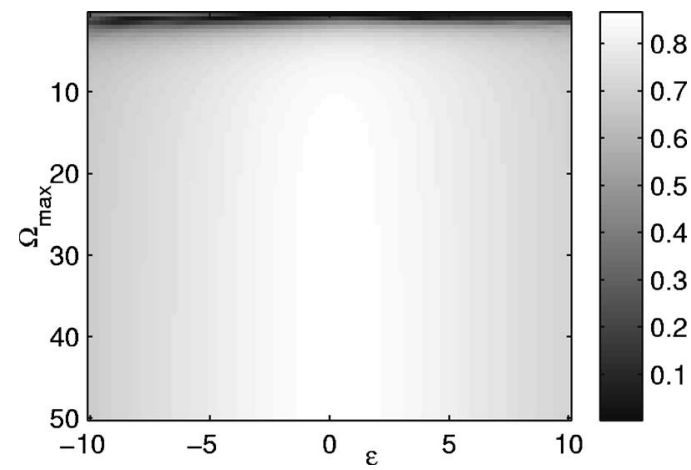

FIG. 5. Conversion efficiency $\eta$ of the feedback control as a function of $\Omega_{\max }$ and $\epsilon$ (in units of $\alpha$ ).

$\Omega_{\max }$ is not too small and the Feshbach detuning $|\epsilon|$ is not too large, compared with the Feshbach coupling strength $\alpha$. The experimental realization of high-efficient atom-molecule conversion is thus feasible with the present feedback STIRAP control scheme.

Finally, we perform sensitivity analysis on the conversion efficiency with respect to the uncertainty of matter interaction parameters. Currently, the scattering lengths of atoms (and therefore the atom-atom collision coefficients such like $\lambda_{a}$ here) are quite well known, but those related to molecules are largely yet to be determined. Let $\lambda_{a}=0.625 \alpha$ be fixed, while $\lambda_{a m}=\lambda_{a g}$ and $\lambda_{m}=\lambda_{g}=\lambda_{m g}$ be two unknown parameters. Now using the dashed-curve in Fig. 3(c) as the control laser detuning $\Delta(t)$, we present in Fig. 6 the evaluated conversion efficiency as a function of these two independent parameters. We see that in the vicinity of the estimated value of $0.1875 \alpha$ for both parameters, the conversion efficiency is more sensitive to the true value of the molecule collision coefficient $\left(\lambda_{g}, \lambda_{m}\right.$, or $\left.\lambda_{m g}\right)$ than its atom-molecule counterpart $\left(\lambda_{a m}\right.$ or $\left.\lambda_{a g}\right)$. This may thus in turn offer the opportunity of utilizing the present adaptive feedback control method to accurately extract the matter system parameters, especially the sensitive ones, from the measured conversion efficiency.

In conclusion, we have presented a feedback STIRAP control method to suppress the dynamical instability in the coherent conversion of an atomic to a molecular Bose-

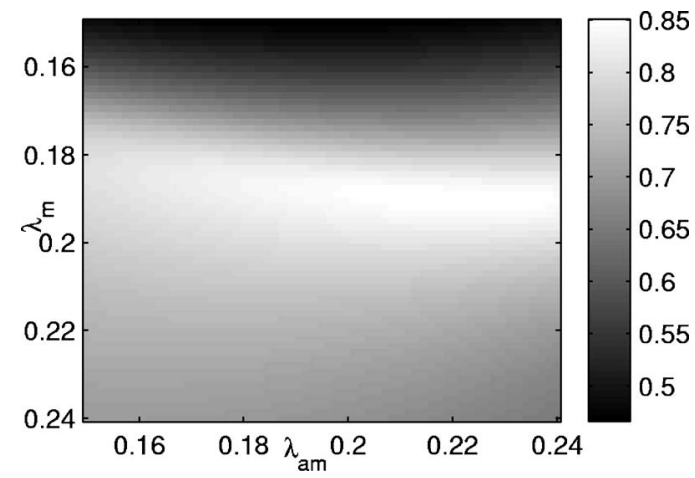

FIG. 6. Effect of the scattering lengths uncertainty on the conversion efficiency. The control field detuning $\Delta(t)$, same as the dashed-curve in 3(c), is determined with the estimated value of $0.1875 \alpha$ for both $\lambda_{m}$ and $\lambda_{a m}$. Assumed are also $\lambda_{g}=\lambda_{m g}=\lambda_{m}$ and $\lambda_{a g}=\lambda_{a m}$. Units of $\lambda_{m}$ and $\lambda_{a m}$ are $\alpha$. 
Einstein condensate. The present work focuses on the case where the atom-molecule coupling is via Feshbach resonance; but the same formulation is applicable to the photoassociation coupling case. The required detuning $\Delta(t)$ [Eq. (4), or frequency chirp] for the adaptive control laser field can be obtained via either the experimental feedback or the propagation of Eqs. (1) with Eq. (4). Unlike previous methods [10-17], the present feedback STIRAP scheme can force the system to stay in CPT states for any parameters. Numerical results indicate that this feedback control method is very robust, and achieves high efficiency of an atom-molecule conversion in realistic systems.

The present work is based on a three-level description of the coupled atom-molecule BEC, which has been widely used in literatures. [12-17]. It has been pointed out that the pairing fields may limit the conversion efficiency in resonantly coupled atom-molecule BECs [18]. Fortunately, this effect seems not important in our STIRAP case since the population in the quasibound state is always very small, thus the number of thermal atoms generated by pairing fields will be also very small. Also we note that beside the STIRAP control scheme, high efficiency molecular population transfer in a linear Hamiltonian system can also be achieved by using shaped laser pulses $[19,20]$. These well-established control schemes in linear Hamiltonian systems for realistic molecules can individually be extended to nonlinear BEC systems. It is also anticipated based on the present work that a feedback control will be needed to overcome the collisioninduced instability. Future work along this direction will include the spatial variations and microscopic interactions in atom-molecule coupling.

Support from the National Natural Science Foundation of China (Grant No. 10404031), Shanghai Rising Star Program, and the Research Grants Council of the Hong Kong Government is acknowledged.
[1] R. A. Duine and H. T. C. Stoof, Phys. Rep. 396, 115 (2004).

[2] E. Timmermans, P. Tommasini, M. Hussein, and A. Kerman, Phys. Rep. 315, 199 (1999).

[3] P. D. Drummond, K. V. Kheruntsyan, and H. He, Phys. Rev. Lett. 81, 3055 (1998).

[4] J. Javanainen and M. Mackie, Phys. Rev. A 58, R789 (1998); Phys. Rev. A 59, R3186 (1999).

[5] R. H. Wynar et al., Science 287, 1016 (2000).

[6] T. Rom et al., Phys. Rev. Lett. 93, 073002 (2004).

[7] D. J. Heinzen, R. Wynar, P. D. Drummond, and K. V. Kheruntsyan, Phys. Rev. Lett. 84, 5029 (2000).

[8] J. J. Hope and M. K. Olsen, Phys. Rev. Lett. 86, 3220 (2001).

[9] Sometimes the obtained molecular state can be a weakly excited bound state based on switching the magnetic field, see M. Mark et al., Europhys. Lett. 69, 706 (2005).

[10] K. Bergmann, H. Theuer, and B. W. Shore, Rev. Mod. Phys. 70, 1003 (1998).

[11] A. Vardi et al., J. Chem. Phys. 107, 6166 (1997).

[12] S. J. J. M. F. Kokkelmans, H. M. J. Vissers, and B. J. Verhaar,
Phys. Rev. A 63, 031601(R) (2001).

[13] M. Mackie, R. Kowalski, and J. Javanainen, Phys. Rev. Lett. 84, 3803 (2000).

[14] M. Mackie, Phys. Rev. A 66, 043613 (2002).

[15] P. D. Drummond, K. V. Kheruntsyan, D. J. Heinzen, and R. H. Wynar, Phys. Rev. A 65, 063619 (2002).

[16] M. Mackie, K. Harkonen, A. Collin, K. A. Suominen, and J. Javonainen, Phys. Rev. A 70, 013614 (2004).

[17] H. Y. Ling, H. Pu, and B. Seaman, Phys. Rev. Lett. 93, 250403 (2004).

[18] M. Holland, J. Park, and R. Walser, Phys. Rev. Lett. 86, 1915 (2001).

[19] J. Cao, C. J. Bardeen, and K. R. Wilson, Phys. Rev. Lett. 80, 1406 (1998); J. Chem. Phys. 113, 1898 (2000).

[20] C. P. Koch, F. Masnou-Seeuws, and R. Kosloff, Phys. Rev. Lett. 94, 193001 (2005); E. Luc-Koenig, R. Kosloff, F. Masnou-Seeuws, and M. Vatasescu, Phys. Rev. A 70, 033414 (2004); C. P. Koch, J. P. Palao, R. Kosloff, and F. MasnouSeeuws, ibid. 70, 013402 (2004). 